\title{
Retrieval of solar magnetic fields from high-spatial resolution filtergraph data: the Imaging Magnetograph eXperiment (IMaX)
}

\author{
D. Orozco Suárez ${ }^{1,2}$, L. R. Bellot Rubio ${ }^{1}$, V. Martínez Pillet ${ }^{3}$, J. A. Bonet ${ }^{3}$, \\ S. Vargas Domínguez ${ }^{4}$, and J. C. del Toro Iniesta ${ }^{1}$
}

1 Instituto de Astrofísica de Andalucía (CSIC), Apdo. Correos 3004, 18080 Granada, Spain e-mail: [lbellot;jti]@iaa.es

2 National Astronomical Observatory of Japan, Mitaka, Tokyo 181-8588, Japan

e-mail: d.orozco@nao.ac.jp

3 Instituto de Astrofísica de Canarias, vía Láctea, s/n, 38200 La Laguna (Tenerife), Spain

e-mail: [vmp; jab]@iac.es

${ }^{4}$ UCL - Mullard Space Science Laboratory, Holmbury St Mary, Dorking, Surrey, RH5 6NT, UK

e-mail: svd@mssl.ucl.ac.uk

Received 8 February 2010 / Accepted 25 June 2010

\section{ABSTRACT}

\begin{abstract}
Context. The design of modern instruments does not only imply thorough studies of instrumental effects but also a good understanding of the scientific analysis planned for the data.

Aims. We investigate the reliability of Milne-Eddington (ME) inversions of high-resolution magnetograph measurements such as those to be obtained with the Imaging Magnetograph eXperiment (IMaX) aboard the SUNRISE balloon. We also provide arguments to choose either Fe I 525.02 or $525.06 \mathrm{~nm}$ as the most suitable line for IMaX.

Methods. We reproduce an IMaX observation using magnetoconvection simulations of the quiet Sun and synthesizing the four Stokes profiles emerging from them. The profiles are degraded by spatial and spectral resolution, noise, and limited wavelength sampling, just as real IMaX measurements. We invert these data and estimate the uncertainties in the retrieved physical parameters caused by the ME approximation and the spectral sampling.

Results. It is possible to infer the magnetic field strength, inclination, azimuth, and line-of-sight velocity from standard IMaX measurements (4 Stokes parameters, 5 wavelength points, and a signal-to-noise ratio of 1000) applying ME inversions to any of the Fe I lines at $525 \mathrm{~nm}$. We also find that telescope diffraction has important effects on the spectra coming from very high resolution observations of inhomogeneous atmospheres. Diffration reduces the amplitude of the polarization signals and changes the asymmetry of the Stokes profiles.

Conclusions. The two Fe I lines at $525 \mathrm{~nm}$ meet the scientific requirements of IMaX, but Fe I $525.02 \mathrm{~nm}$ is to be preferred because it leads to smaller uncertainties in the retrieved parameters and offers a better detectability of the weakest (linear) polarization signals prevailing in the quiet Sun.
\end{abstract}

Key words. magnetic fields - Sun: photosphere - instrumentation: high angular resolution

\section{Introduction}

With the advent of new instrumentation, we are witnessing a true revolution in our understanding of the quiet-Sun magnetism. The combination of superb spatial resolution and high-precision polarimetry has unveiled a panorama in which internetwork regions appear to be covered by very inclined, weak magnetic fields that had hitherto been elusive to observation. These findings have been possible thanks to an increase in the spatial resolution of spectropolarimetric measurements. Resolutions better than, say, 0.'5 were hardly reachable with ground-based polarimeters just five years ago. Since 2006, however, the Hinode spectropolarimeter (SP, Lites et al. 2001; Kosugi et al. 2007; Tsuneta et al. 2008), is providing data with spatial resolutions of 0.32 on a regular basis, with a signal-to-noise ratio (SNR) of about 1000. The analysis of these measurements has shown that the weak internetwork fields occupy fractions of 20-45\% of the SP pixel (Lites et al. 2007, 2008; Orozco Suárez et al. 2007; Orozco Suárez et al., in prep.; Orozco Suárez 2008). Thus, we are nearly resolving the magnetic fields that form the internetwork, in the sense that the filling factor is approaching $100 \%$. But these fields are not yet directly visible with current instruments and their properties have to be determined by means of careful analyses.

Further improvements in the spatial resolution will conceivably lead to new discoveries. Since the polarization signals scale linearly with the filling factor, they will become larger if the internetwork is unevenly permeated by magnetic structures not much smaller than the current resolution limit. On the contrary, if the characteristic sizes are very small and the structures homogeneously distributed, then the observed polarization amplitudes will not increase at higher spatial resolution because the amount of cancellation of opposite polarity signals will remain the same ${ }^{1}$. There might be a third possibility that the magnetic structures are small but fractally distributed (Pietarila Graham et al. 2009). In this case, the cancellation of opposite polarity

\footnotetext{
1 Traditionally, the cancellation of Zeeman signals has been associated with the presence of mixed-polarity fields at the spatial resolution of several arcsec achieved by Hanle measurements of the quiet Sun (see, e.g., Trujillo Bueno et al. 2004).
} 
Table 1. Basic parameters of the SunRISE/IMaX system.

\begin{tabular}{lr}
\hline \hline TELESCOPE APERTURE & $1 \mathrm{~m}$ \\
TELESCOPE CENTRAL OBSCURATION & $35.2 \%$ \\
WORKING WAVELENGTH & $525.02 \mathrm{~nm}$ \\
SPATIAL RESOLUTION & $\sim 0^{\prime} \cdot 11$ \\
CCD PIXEL SIZE & $0.0^{\prime} 055 \times 0^{\prime} 055$ \\
IMaX PASSBAND $(F W H M)$ & $\sim 6 \mathrm{pm}$ \\
\hline
\end{tabular}

signals has been observed to depend on the spatial resolution at least down to the 0.3 of Hinode ${ }^{2}$. The subject is currently feeding an intense debate. A distinction between the scenarios and the determination of their relative contributions to the total magnetic flux is very important and can effectively be achieved by using larger telescopes and advanced techniques for high resolution spectropolarimetry.

In moments of very good seeing, the CRisp Imaging SpectroPolarimeter (CRISP, Scharmer 2006), installed at the Swedish Solar Telescope on La Palma (Spain), deliver polarimetric images near the diffraction limit of its 1-m telescope with the help of adaptive optics and computationally-expensive image restoration techniques (van Noort et al. 2005). CRISP is a filter-based instrument, which allows the observation of large fields of view while preserving high spatial resolution and polarimetric accuracy. Compared to spectrograph-based polarimeters, filter instruments also have drawbacks like poorer spectral resolution and wavelength sampling. Several lines can be observed sequentially, but at the expense of slower cadences (without degrading the SNR). These advantages and disadventages imply differences in the results obtained with each instrument that deserve attention, especially when a new one is designed and constructed. The way the instrument treats the polarization signals, the pointing stability, the wavelength samples, the magnetic sensitivity of the selected spectral line, the polarization modulation scheme, the polarimetric efficiency, and the influence of environmental (instrumental plus atmospheric) polarization are some of the issues to be considered.

The Imaging Magnetograph eXperiment (IMaX) is a dualbeam vector spectropolarimeter based on a Fabry-Pérot étalon as the spectrum analyzer and a pair of liquid crystal variable retarders as the polarization modulator (Martínez Pillet et al. 2010; see also Jochum et al. 2003; Martínez Pillet et al. 2004; and Álvarez-Herrero et al. 2006). IMaX is one of the postfocus instruments of the SUNRISE 1m telescope, which flew over the Artic in a stratospheric balloon from June 8 to 13, 2010 (Barthol et al. 2010). In this way, continuous observations of the Sun were possible avoiding most of the Earth atmosphere. Table 1 summarizes the basic parameters of the instrument. IMaX provides capabilities for obtaining polarimetric images near the diffraction limit of SUNRISE ( 0.11 at $525 \mathrm{~nm}$ ), with a SNR of about 1000 . The instrument has several observing modes in which the number of wavelengths is varied from 3 through 12 (one of them is always reserved for the continuum), but the "regular", so-called vector spectropolarimetric, mode is one that scans the line at 5 wavelength points in $33 \mathrm{~s}$. Will it be possible to accurately infer the full vector magnetic field from the regular IMaX measurements using appropriate inversion techniques? Answering this question is the main aim of the present paper.

To assess the accuracy to which the magnetic field strength, inclination, azimuth, and line-of-sight (LOS) velocity can be

2 This effect was previously shown in simulations by Sánchez Almeida et al. (2003). determined, we here simulate an IMaX observation. We use radiative magnetohydrodynamic (MHD) models to synthesize the Stokes profiles of the photospheric Fe I lines at 525.02 and $525.06 \mathrm{~nm}$. These two lines, very close in wavelength and hence observable with the same pre-filter, were considered for the IMaX instrument since the beginning of the design phase. In fact, part of the results of this paper provided the rationale for selecting one of the lines instead of the other. An earlier investigation on magnetic inferences from filtergrams with limited wavelength sampling was carried out by Graham et al. (2002). They used Milne-Eddington (ME) profiles to simulate the observations. Our paper can be considered as an extension of their work, since MHD simulations provide a more realistic description of the solar photosphere.

The simulated Stokes profiles are degraded by telescope diffraction and detector pixel size to a spatial resolution of $80 \mathrm{~km}$ on the solar surface, and then sampled at five wavelengths (four within the line plus another in the nearby continuum) to mimic the nominal vector spectropolarimetric mode of IMaX. From these data we determine the magnetic field vector and LOS velocity using ME inversions and compare them with the parameters resulting from the inversion of fully resolved and critically sampled profiles. To simulate a real observation, the typical noise of IMaX measurements is added to the profiles.

The paper is structured as follows: Sect. 2 describes the spatial degradation of the simulated Stokes profiles and the effects of the tunable filter on the spectra. In that section we also analyze the effects of noise and the drawbacks of selecting only a few wavelength positions across the line. Section 3 deals with the results of ME inversions. There we estimate the uncertainties of the retrieved physical parameters. In Sect. 4 we summarize the main conclusions of this work.

\section{Simulating IMaX observations}

\subsection{Spatial degradation}

To simulate an IMaX observation we use model atmospheres from the radiative MHD calculations of Vögler et al. (2005). They were initialized with a distribution of mixed polarity fields with an average magnetic field strength of $\langle B\rangle \sim 200 \mathrm{G}$ at $\log \tau=-1$. The snapshot used here was taken when $\langle B\rangle$ had decayed to about $\sim 140 \mathrm{G}$. We have chosen this particular run because it contains both network- and internetwork-like regions. The extent of the computational box is $288 \times 288 \times 100$ pixels, with a spatial sampling of $20.8 \mathrm{~km}\left(0{ }^{\prime} 0287\right)$ in the horizontal direction and $14 \mathrm{~km}$ in the vertical direction. The bottom of the box is located $800 \mathrm{~km}$ below $\tau=1$. More details about this run can be found in Khomenko et al. (2005).

Since the work of Vögler et al. (2005), simulations of higher numerical resolution have become available. They certainly represent a significant step forward, but we believe they would not change our conclusions, at least for the larger magnetic structures present in the network and most of the internetwork - the ones observed by IMaX during its first flight and also by Hinode since its launch in 2006. The reason is that the simulations of Vögler et al. (2005) have been confronted with observations thoroughly with excellent results. This indicates that they already provide a very good description of the solar atmosphere.

The MHD models are used to generate the observations by synthesizing the Stokes $I, Q, U$, and $V$ profiles with the SIR code (Ruiz Cobo \& Del Toro Iniesta 1992). The spectral synthesis is performed in a wavelength range that spans $1 \mathrm{~nm}$ 

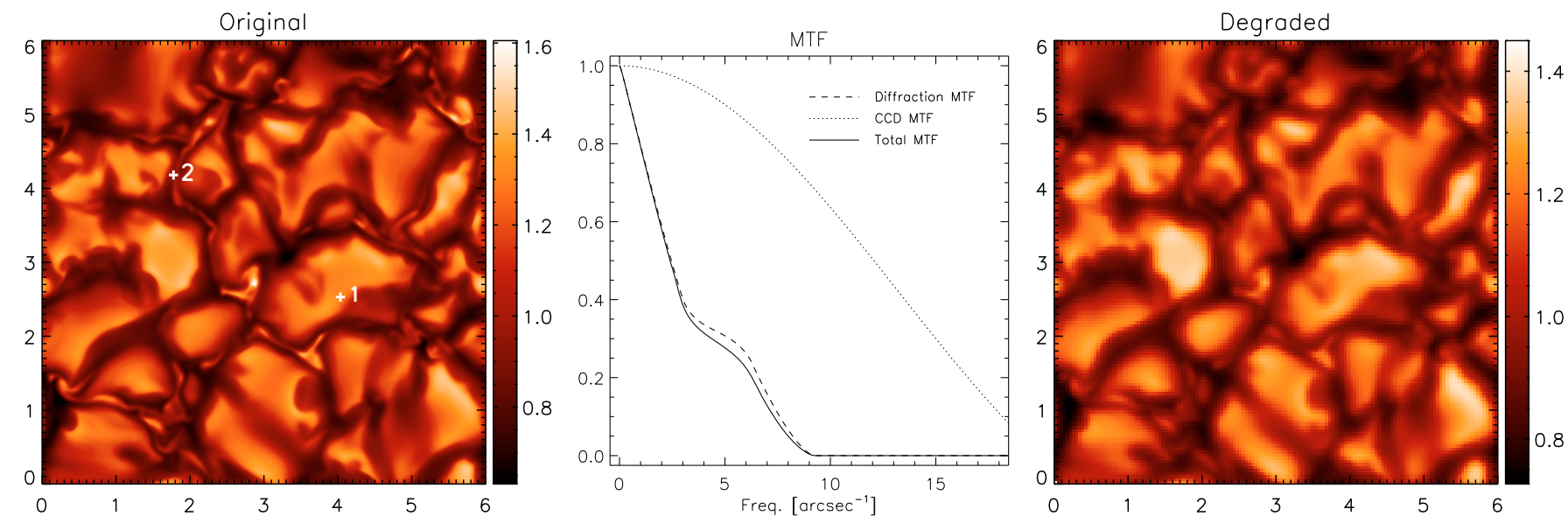

Fig. 1. Continuum intensity map at the original resolution of the MHD models (left) and degraded considering telescope diffraction and detector pixel size (right). The central panel shows the MTF of the CCD (dotted line), that of the diffraction-limited telescope including the central obscuration (dashed line), and the total MTF (solid line).

and includes the Fe I lines at 525.02 and $525.06 \mathrm{~nm}$. The wavelength step is $1 \mathrm{pm}$. Next, the monochromatic images are spatially degraded as the SUNRISE/IMaX system does. The modulation transfer function (MTF) of the whole system is depicted in Fig. 1 and includes the $1 \mathrm{~m}$ aperture of the telescope, the central obscuration of the entrance pupil (caused by the secondary mirror), and the effects of image pixelation. The spatially degraded images are binned by a factor 2 , hence their sampling is almost identical to that of IMaX with a pixel size of 0. '055. The original continuum contrast of $17.9 \%$ decreases only by $4 \%$ due to diffraction and CCD pixelation. After that we convolve the Stokes profiles with a Gaussian of $6 \mathrm{pm} F W H M$ to account for the limited spectral resolving power of the FabryPérot étalon. Finally, we add noise and select four wavelength samples across the line plus a wavelength point in the nearby continuum.

The spatial degradation described above represents a rather pessimistic estimate because IMaX took in-flight calibrations of the optical aberrations for post-facto restorations. This will increase the power at all frequencies up to the cut-off limit. The calibration procedure (Vargas Domínguez 2008) is based on the phase diversity (PD) technique for image restoration and wavefront sensing (Paxman et al. 1996). The instrument has a glass plate that can be optionally intercalated between the beam splitter and one of the CCDs to induce a controlled defocus. Therefore, a simultaneous focus-defocus image pair (PD pair) can be recorded by employing the two cameras. From this pair, an estimate of the wavefront aberration, parameterized in the form of a Zernike polynomials expansion, will be possible during the data processing by means of a PD inversion code. Deconvolution of the derived point spread function of the system will be carried out by Wiener filtering the data. Since this will partially correct the effects of the system, our using the theoretical MTF shown in Fig. 1 means we are considering a worst-case scenario.

The two most important effects of telescope diffraction are spatial blurring and Stokes profile smearing. The first takes place because (polarized) light spreads from every pixel to its immediate surroundings. As a consequence, the spatial resolution decreases, the polarization amplitudes tend to diminish, and the signals are more vulnerable to photon noise. In addition, the spatial resolution decreases. Figure 2 displays monochromatic Stokes $Q, U$ and $V$ images at $+7.7 \mathrm{pm}$ from the center of the Fe I $525.02 \mathrm{~nm}$ line where these effects can be observed. To emphasize details we represent a small area of the simulation snapshot. (A) stands for the images at original resolution and (B) for the images spatially degraded by telescope diffraction and CCD pixelation. We have added noise at the level of $10^{-3} I_{\mathrm{c}}$ in order to see how it affects the observations. Since Stokes $Q$ and $U$ have weaker signals than Stokes $V$, they are significantly more degraded than the latter. In general, the images do not look strongly altered; the polarization amplitudes decrease and some structures escape detection, but the morphological aspect is essentially preserved.

The second effect comes from the fact that the spatially averaged Stokes profiles are less asymmetric. As an example, we show in Fig. 3 the effect of pure spatial degradation (telescope plus detector) on the polarization profiles. The Stokes $I$ and $V$ signals from two different points (indicated by numbers 1 and 2 in Fig. 1) are displayed. Note the strong modification of the continuum and the shape of the profiles, prior to any action of the IMaX étalon. Similar modifications can be expected from any instrument, but apparently they have not been described in the literature. MHD simulations like the ones we are using here are the best means to date to bring this effect to the attention of the community: details of the profiles are lost because of the finite spatial resolving power.

\subsection{Spectral degradation}

IMaX uses a single Fabry-Pérot étalon in double pass for the spectral analysis. The $F W H M$ of the IMaX spectral point spread function (PSF) is $\sim 6 \mathrm{pm}$. For simplicity, we approximate the shape of the PSF by a Gaussian function. The effect of the IMaX étalon on the polarization images is shown in column (C) of Fig. 2. Naturally, the amplitudes diminish and the weakest signals disappear below the noise.

As is well known, the spectral smearing induced by the étalon smooths out the profile shapes and the Stokes spectra become more symmetric. Therefore, some of the weakest signals, typically linear polarization signals, cannot be detected after the combined action of the telescope, the étalon, and the detector. But the problem is not only on detection. These effects also reduce the information content of the detected profiles. The sensitivity to gas velocities, for example, decreases because the étalon broadens the profiles and makes them shallower (Cabrera Solana et al. 2005). The sensitivity to other atmospheric 
(A)
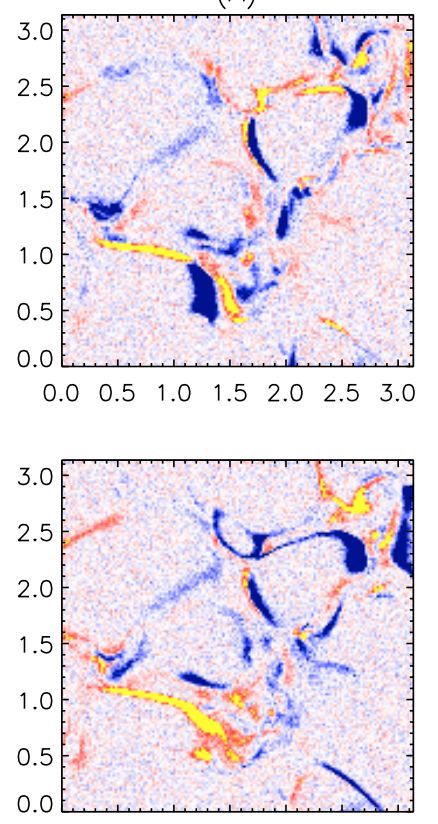

$\begin{array}{lllllll}0.0 & 0.5 & 1.0 & 1.5 & 2.0 & 2.5 & 3.0\end{array}$

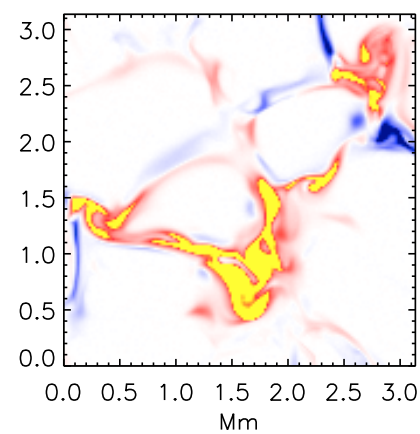

(B)
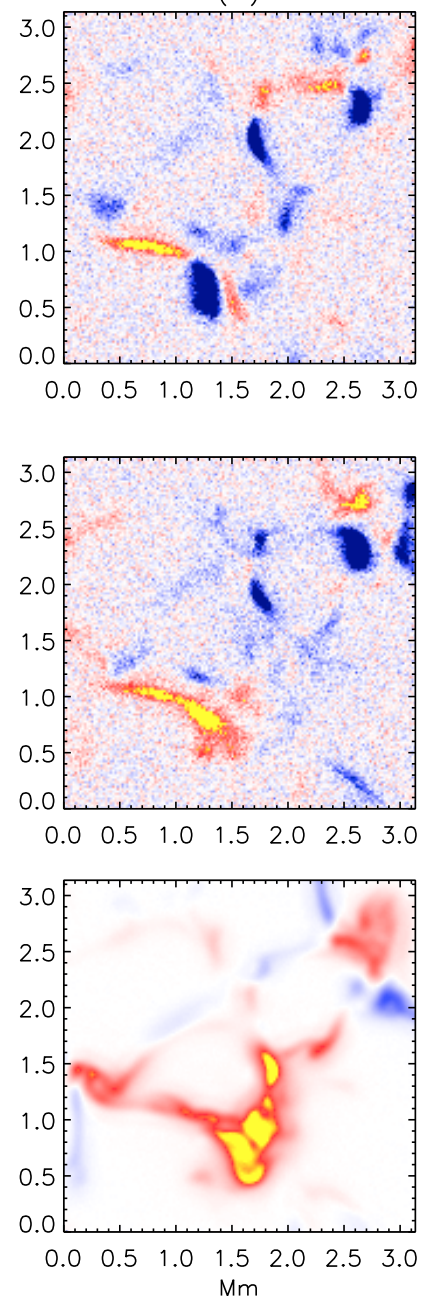

(C)
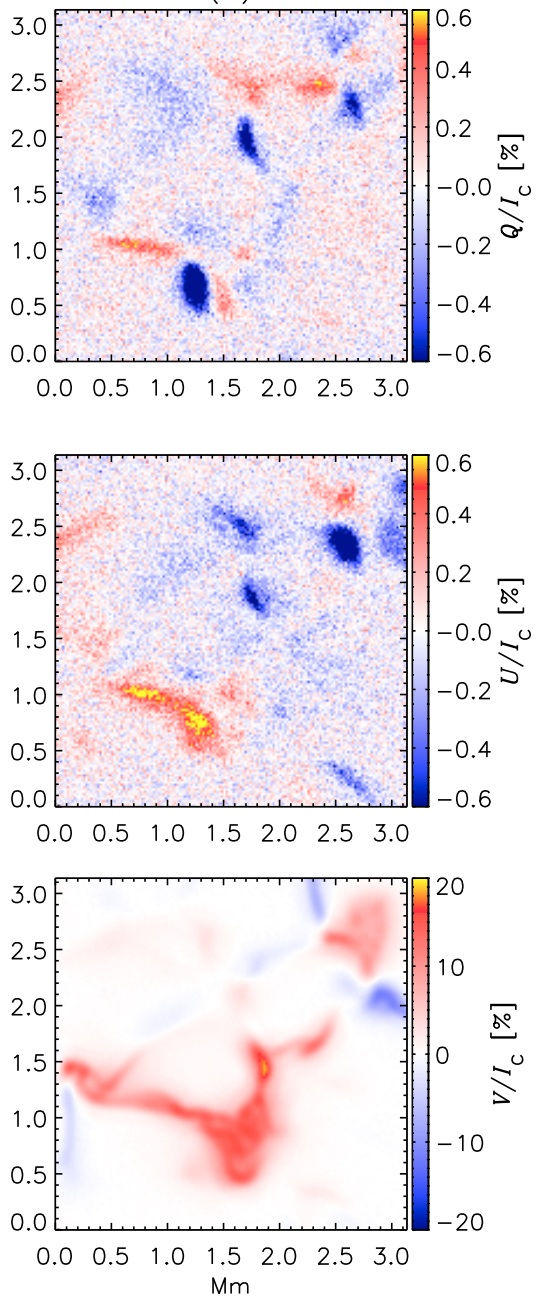

Fig. 2. Monochromatic Stokes $Q$ (top), $U$ (middle), and $V$ (bottom) images at $+7.7 \mathrm{pm}$ from the central wavelength of the Fe I line at $525.02 \mathrm{~nm}$. From left to right: the original data (A), the spatially degraded data (B), and the spatially and spectrally degraded data after taking into account the spectral PSF of $6 \mathrm{pm}(\mathrm{C})$. Only a region of $150 \times 150$ pixels is displayed.

parameters also decreases with a wider spectral PSF because response functions also get broadened by it (Orozco Suárez \& Del Toro Iniesta 2007).

\subsection{The effect of noise}

Both telescope diffraction (plus CCD pixelation) and spectral analysis reduce the amplitude of the polarization signals. Consequently, they are more affected by the noise. In this section we quantify the influence of photon noise on the measurements.

Figure 4 shows the percentage of pixels whose Stokes $Q$ or $U$ amplitudes exceed specified noise levels. The Fe I lines at 525.06 and $525.02 \mathrm{~nm}$ are represented in red and black, respectively. Obviously, the amount of detectable linear polarization signal decreases quite rapidly with noise. Noise levels of $3 \times 10^{-3} I_{\mathrm{c}}$ and $10^{-3} I_{\mathrm{c}}$, the latter corresponding to the IMaX case, are marked with vertical lines. The dotted lines stand for the spatially degraded Stokes profiles and the solid lines for the spatially and spectrally degraded profies. $80 \%$ and $55 \%$ of the pixels show linear polarization signals in Fe I $525.02 \mathrm{~nm}$ and $525.06 \mathrm{~nm}$ that exceed the noise level before the light enters the instrument. These values change to $50 \%$ and $20 \%$ after the spectral analysis. Interestingly, the final detectability of linear polarization in the $525.02 \mathrm{~nm}$ line is almost the same as that of $525.06 \mathrm{~nm}$ before the spectral analysis. Thus, Fe I $525.02 \mathrm{~nm}$ presents clear advantages for detecting weak linear polarization signals in the quiet Sun.

Figure 5 shows histograms of the Stokes $V$ amplitudes in the original maps (solid lines), the spatially degraded maps (dashed lines), and the spatially plus spectrally degraded maps (dashdotted lines), for Fe I 525.06 and $525.02 \mathrm{~nm}$ (top and bottom, respectively). The histograms show an asymmetric distribution of amplitudes with two peaks corresponding to network and internetwork points, the latter being responsible for the smaller $V$ amplitudes. The histograms for the $525.02 \mathrm{~nm}$ line are shifted to stronger signals because of the enhanced sensitivity of this line to magnetic fields. Again, specific noise levels are indicated by the vertical lines. In the original data, the percentage of pixels with Stokes $V$ amplitudes below $10^{-3} I_{\mathrm{c}}$ is $0.06 \%$ and $0.5 \%$ for the 525.02 and $525.06 \mathrm{~nm}$ lines, respectively. These percentages change to $1.6 \%$ and to $4.3 \%$ when the profiles are degraded spatially and spectrally ${ }^{3}$. Therefore, most of the $V$ signals would be observable with our instrument if we use the $525.02 \mathrm{~nm}$ line and the SNR is 1000. Thus, Fe I $525.02 \mathrm{~nm}$ reveals itself

${ }^{3}$ Of course, if $S N R=300$, the Stokes $V$ detectability decreases: the percentages reach $2 \%$ and $5.3 \%$ in the original data, and $11.4 \%$ and $23.2 \%$ after degradation. 
D. Orozco Suárez et al.: Retrievals from high-spatial resolution filtergraph data
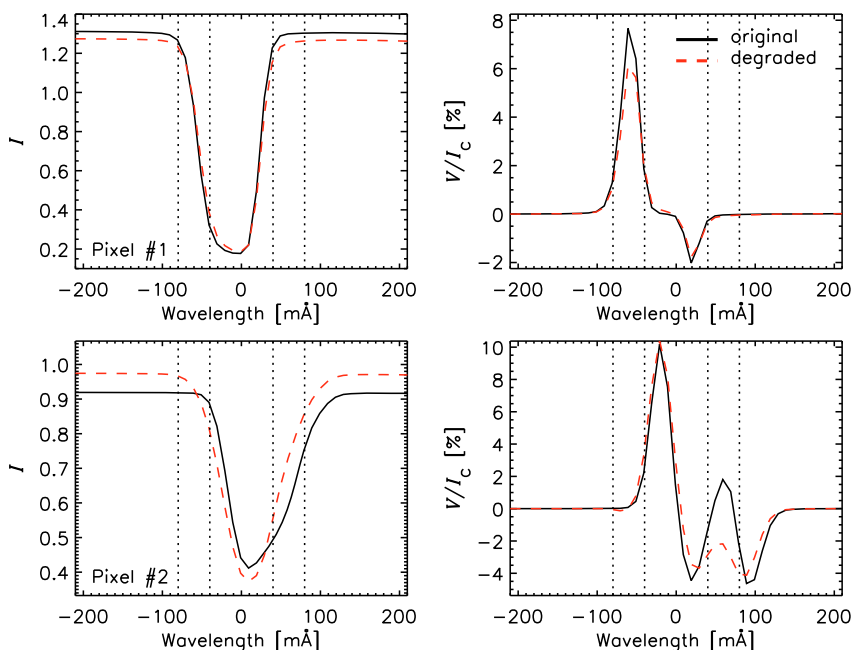

Fig. 3. Stokes $I$ and $V$ profiles of Fe I $525.02 \mathrm{~nm}$ corresponding to pixels \#1 (top) and 2 (bottom) in Fig. 1. The solid and dashed lines represent the original simulations and the maps degraded by telescope diffraction and CCD pixelation, respectively. The vertical lines indicate wavelength samples at $[ \pm 8, \pm 4] \mathrm{pm}$ from the central wavelength of the line. To make a pixel-to-pixel comparison, we have taken the Stokes profiles from the degraded data before binning the images to match the IMaX CCD pixel size. Therefore, dissimilarities are enhanced.

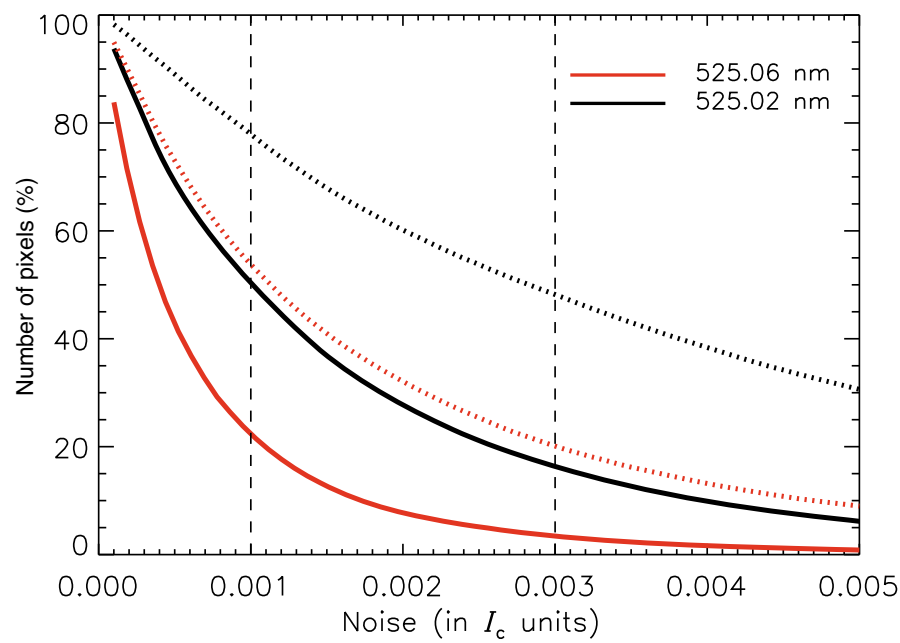

Fig. 4. Variation in the percentage of pixels where either Stokes $Q$ or $U$ exceed a given noise level. The dotted and solid lines correspond to data before and after convolution with the spectral PSF. The vertical lines represent noise levels of $10^{-3}$ and $3 \times 10^{-3} I_{\mathrm{c}}$.

as the better choice for detecting both weak linear and circular polarization signals.

Further degradation is expected due to the limited wavelength sampling. As mentioned in the introduction, the regular vector spectropolarimetric mode of IMaX measures the polarization state in 4 wavelength within the line plus another in the continuum. These wavelengths have been marked with vertical lines in Fig. 3. The choice of a limited wavelength sampling is not arbitrary: on the one hand, scanning the line at just a few points reduces the scan time, improving the temporal integrity of the data and their immunity to variations in the conditions of the dynamic Sun or changes in the solar scene; on the other hand, a better sampling was not needed because full line profiles were going to be obtained with the slit spectropolarimeter originally included in the scientific payload of SUNRISE. Here we
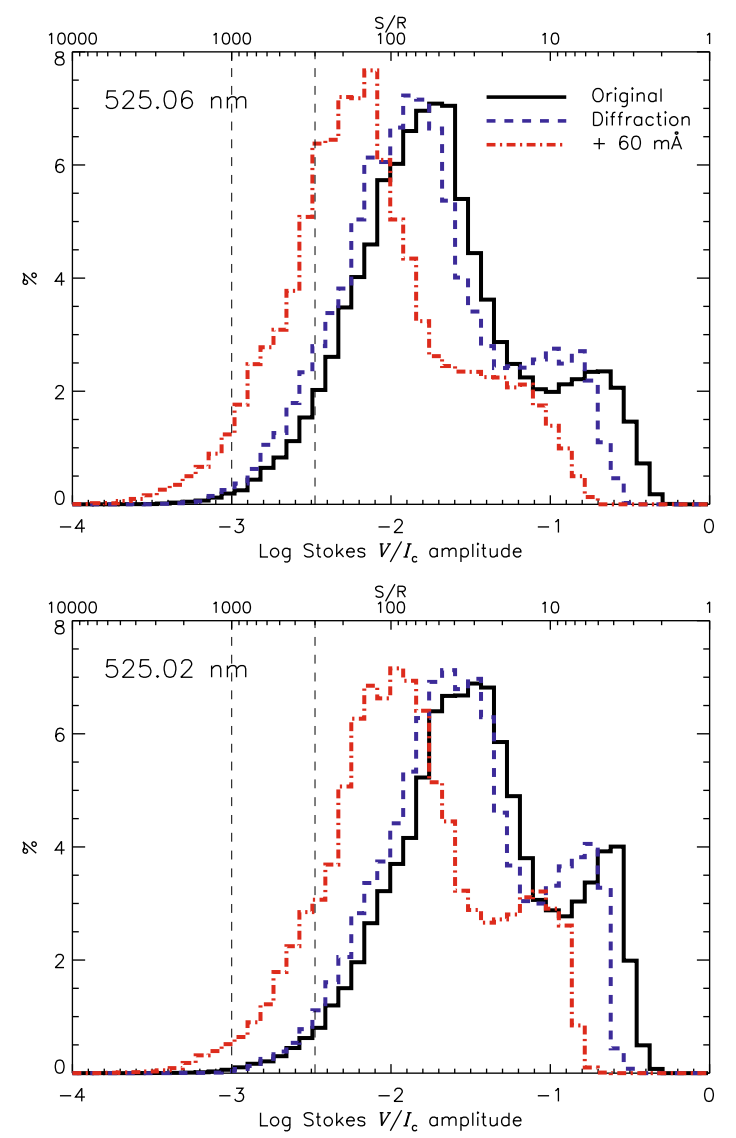

Fig. 5. Distribution of the Stokes $V / I_{\mathrm{c}}$ amplitudes in the original, spatially and spectrally degraded data. The vertical lines represent noise levels of $10^{-3}$ and $3 \times 10^{-3} I_{c}$.

are concerned with the regular IMaX spectropolarimetric mode and therefore we have to acknowledge a further decrease in the fraction of polarization signals detectable above the noise level.

\section{Inversion of the Stokes profiles}

In this section we address two questions. First, we want to know which line is less affected by the constraints imposed by the instrument (i.e., spectral degradation, limited wavelength sampling, photon noise). The answer to this question is important to choose the most suitable line for IMaX. Second, we want to determine the uncertainties that can be expected from the analysis of the measurements taken in the selected line.

To answer the first question, we need to evaluate the influence of instrumental effects on the ME parameters resulting from the inversion of the two spectral lines. This requires a differential analysis. In principle, since the original model atmospheres are at our disposal, it would be straightforward to compare the inversion results for each pixel and each spectral line as observed by IMaX with the corresponding MHD models. However, such an absolute analysis would not inform us about the relative merits of the two lines. The reason is that they are formed in slightly different layers of the atmosphere, and a direct comparison of the ME parameters derived from them with the MHD parameters at a fixed height would yield differences that do not reflect the capabilities of the lines, but rather their different formation heights.

The best way to solve this problem is to set up a reference for each of the lines. Here, the reference is taken to be the result of a 

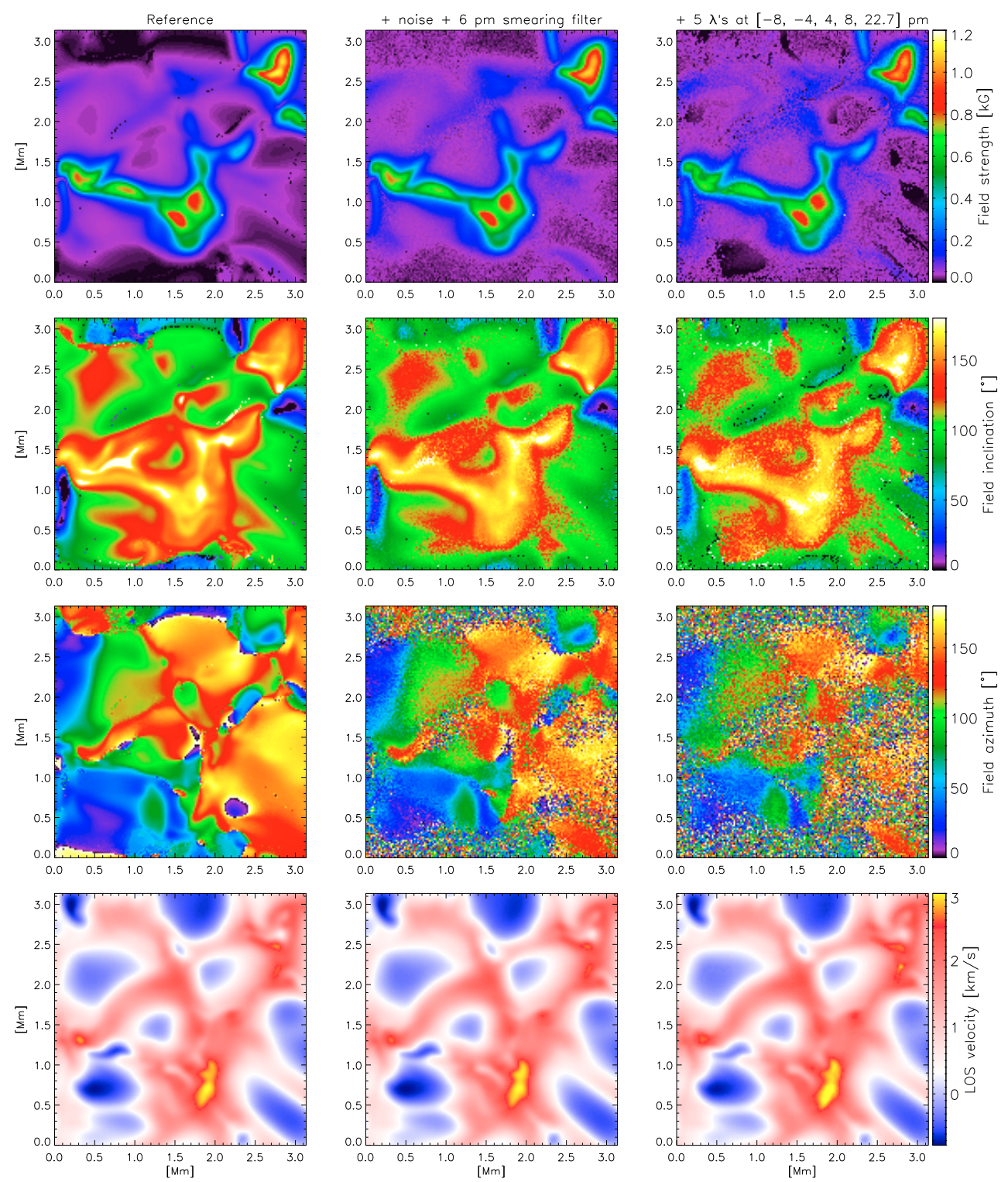

Fig. 6. ME inversions of the Fe I $525.02 \mathrm{~nm}$ line synthesized with the help of MHD models. From top to bottom: magnetic field strength, inclination and azimuth, and LOS velocity. The left panels represent the results from the data degraded by telescope diffraction and CCD pixelation. The middle panels show the maps retrieved from the degraded profiles after convolution with a $6 \mathrm{pm}$ wide filter and addition of noise. The right panels display the results obtained from the spatially and spectrally degraded observations with noise, sampled at five wavelengths points. Except for the reference, $S N R=1000$ in all maps.

ME inversion of the spatially degraded Stokes profiles without noise, sampled at 61 wavelength points separated by $1 \mathrm{pm}$. They represent the idealized case in which the instrument would measure fully resolved Stokes profiles with excellent (infinite) signal-to-noise ratios. Note that the reference includes the effects of telescope diffraction, whose influence on the polarization spectra has already been analyzed in Sect. 2 .

The differential analysis is performed by inverting the simulated IMaX measurements in the two lines and comparing the inferred ME parameters with the corresponding reference. The results, presented in Sect. 3.1, led to the selection of Fe I $525.50 \mathrm{~nm}$ as the IMaX line.

Answering the second question requires a comparison of the original MHD models and the ME parameters derived from the Fe I $520.52 \mathrm{~nm}$ measurements. This is done in Sect. 3.2 below.
All the inversions are carried out assuming a simple onecomponent model atmosphere and no stray-light contamination. The magnetic field vector is initialized with $B=200 \mathrm{G}, \gamma=20^{\circ}$, and $\chi=20^{\circ}$. The inversion code used in the tests is MILOS (Orozco Suárez \& Del Toro Iniesta 2007).

\subsection{Impact of instrumental effects}

To determine the deviations induced by the instrument with respect to the reference, we invert the simulated IMaX data, i.e., the spatially degraded Stokes profiles, smeared by a spectral PSF of $6 \mathrm{pm} F W H M$, sampled at 5 wavelength points (one of which is continuum), and with noise added at the level of $10^{-3} I_{\mathrm{c}}$.

The inversion returns the three components of the vector magnetic field and the LOS velocity. The maps corresponding to the Fe I $525.02 \mathrm{~nm}$ line are displayed in Fig. 6. The left 

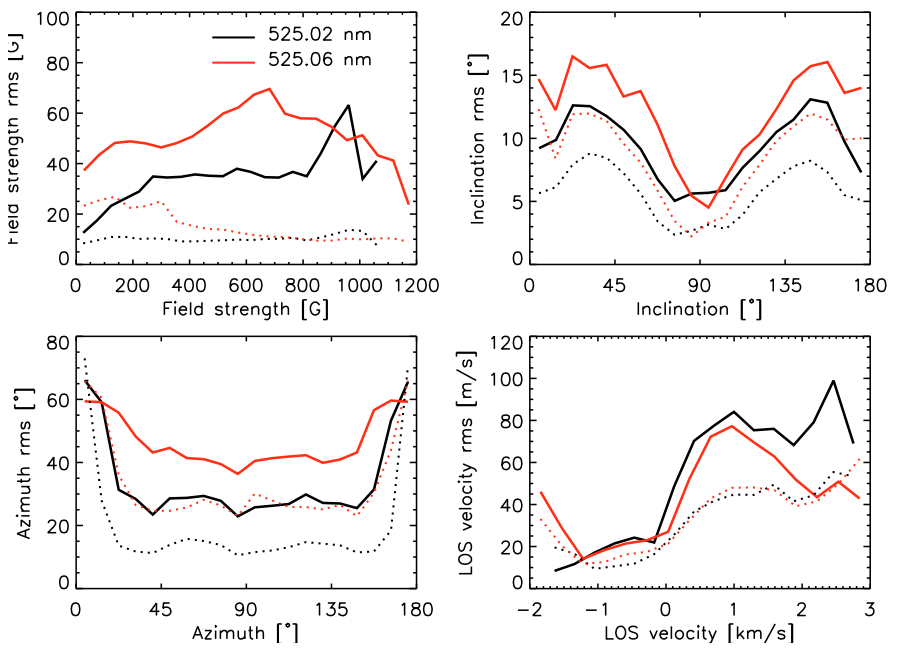

Fig. 7. Rms differences between the inferred ME parameters and the reference. The black and red lines stand for Fe I $525.02 \mathrm{~nm}$ and $525.06 \mathrm{~nm}$, respectively. The dotted lines correspond to the inversion of the degraded profiles but with full spectral sampling. The solid lines show the results for the degraded data sampled at the five wavelengths of the IMaX vector spectropolarimetric mode.

column shows the reference. The middle column displays the results of inverting the profiles convolved with a PSF of $6 \mathrm{pm}$ FWHM and with noise, but still sampled at 61 wavelengths. The right column gives the results obtained from the simulated IMaX measurements. Only a region corresponding to $150 \times 150$ pixels is displayed.

A comparison of the maps demonstrates that the field strength and the LOS velocity are well recovered. Only small deviations from the reference parameters can be seen, although the deviations increase as the field weakens (especially below $100 \mathrm{G})$. The inclination and azimuth maps show the largest deviations because the spectral smearing, the noise, and the limited sampling reduce the Stokes $Q$ and $U$ amplitudes. The noisier areas in the maps derived from the simulated IMaX data correspond to regions where the linear polarization signals are buried in the noise and the field is very weak.

The overall conclusion drawn from these tests is that ME inversions of IMaX measurements in the Fe I $525.02 \mathrm{~nm}$ line are reliable. The accuracy can be expected to be higher for the field strength and the LOS velocity, but also the magnetic inclination and azimuth, although noisier, will be recovered reasonably well.

To support this idea more quantitatively, Fig. 7 displays the rms deviations between the ME parameters inferred from each line and the corresponding reference. The rms values have been calculated by dividing the $x$-axis in bins of size $50 \mathrm{G}, 9^{\circ}$, and $300 \mathrm{~m} \mathrm{~s}^{-1}$. The black and red lines refer to Fe I 525.02 and $525.06 \mathrm{~nm}$, respectively. The solid lines indicate the results obtained from the spatially and spectrally degraded observations sampled at the five wavelength positions of IMaX (the corresponding maps are the ones in the right column of Fig. 6). The rms values refer only to pixels in which the Stokes $Q, U$ or $V$ signals of Fe I $525.06 \mathrm{~nm}$ exceed three times the noise level, except for the LOS velocity where we included all the pixels. Clearly, the $525.02 \mathrm{~nm}$ line yields smaller deviations; typical values are $35 \mathrm{G}$ for the field strength, $10^{\circ}$ and $35^{\circ}$ for the magnetic inclination and azimuth, and $50 \mathrm{~m} \mathrm{~s}^{-1}$ for the LOS velocity. It is worth noting that the deviations caused by the

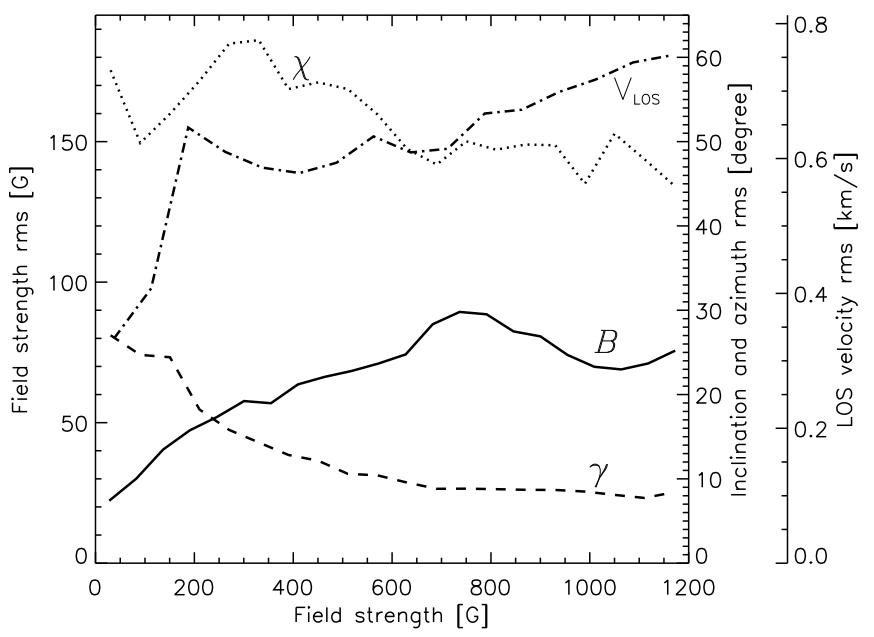

Fig. 8. Rms differences between the ME parameters inferred from the inversion of the Fe I line at $525.02 \mathrm{~nm}$ as observed by IMaX and the corresponding MHD parameters at an optical depth of $\log \tau=-1.5$. The different line shapes stand for different ME parameters: solid - magnetic field strength, dashed - field inclination, dotted - field azimuth, and dotdashed - LOS velocity.

limited spectral sampling are at least twice as large as those associated with instrumental degradation effects (dotted lines in Fig. 7; see also the middel panels of Fig. 6).

In summary, the results show that both lines are suitable for magnetic field and velocity determinations based on $\mathrm{ME}$ inversions, although the deviations may be large for the field inclination and azimuth due to the weakness of the linear polarization signals. Among the two, however, the line at $525.02 \mathrm{~nm}$ is to be preferred because of its larger signals and smaller deviations.

\subsection{Uncertainties of the IMaX spectral line}

The total uncertainties expected for the ME parameters inferred from the IMaX vector spectropolarimetric mode with the Fe I line at $525.02 \mathrm{~nm}$ can be calculated directly by comparing the inversion results with the MHD models. Figure 8 shows the standard deviation of the differences between the ME parameters and the corresponding MHD values at an optical depth ${ }^{4}$ of $\log \tau=-1.5$. Since an IMaX pixel is equivalent to four pixels in the MHD simulations, the latter have been binned by a factor of two in both directions to allow comparisons. For the magnetic field strength, the rms values are never larger than $100 \mathrm{G}$. For the field inclination, the uncertainty increases as the field weakens, reaching a maximum of $\sim 30^{\circ}$. The rms values for the field azimuth are larger, on the order of $30-40^{\circ}$. In the case of the LOS velocity, the rms decreases for weak fields from 0.75 down to $0.35 \mathrm{~km} \mathrm{~s}^{-1}$.

There are three sources of error contributing to the values shown in Fig. 8: the uncertainties caused by the instrumental trade-offs (spectral smearing, wavelength sampling, and noise) as given in Fig. 7, the uncertainties associated with the spatial degradation, and the limitations of the ME approximation. The rms differences displayed in Fig. 8 are largely dominated by the limitations of the ME analysis.

\footnotetext{
${ }^{4}$ We have chosen the optical depth leading to the smaller rms differences.
} 
A detailed study of the uncertainties associated with ME inversions can be found in Orozco Suárez et al. (2010). These authors analyzed noise-free synthetic observations computed from the same MHD models we use here, assuming very high spectral resolution and supercritical wavelength sampling ${ }^{5}$. They concluded that ME inversions provide atmospheric parameters that are averages of the actual parameters along the line of sight. Their results show that the uncertainties due to the ME approximation are larger than those associated with photon noise.

\section{Summary and conclusions}

We have presented an analysis of the reliability of MilneEddington inversions applied to high resolution magnetograph observations such as those delivered by IMaX. The analysis was indeed meant to help during the design and development phases of the instrument. In particular, the results of this work enabled the IMaX team to select the Fe I line at $525.02 \mathrm{~nm}$ instead of Fe I $525.06 \mathrm{~nm}$. Both lines could be used through the IMaX pre-filter, but the former permits more accurate inferences of the vector magnetic field. Fe I 525.02 is also advantageous for the detection of the weakest linear polarization signals of the quiet Sun.

Our analysis relies on synthetic Stokes spectra emerging from realistic MHD simulations of the quiet Sun. The spectra were degraded by taking into account all the SUNRISE/IMaX instrumental effects, including telescope diffraction and CCD pixelation, smearing by the spectral PSF of the FabryPérot étalon, and coarse spectral sampling (the IMaX vector spectropolarimetric mode scans the polarization profiles at just four wavelengths within the line and one continuum point). Finally, the simulated IMaX observations were inverted in terms of Milne-Eddington atmospheres.

Our main conclusion is that ME inversions can provide reasonably accurate atmospheric parameters (especially the magnetic field strength and the LOS velocity) from the resolved but coarsely sampled Stokes spectra acquired by SUNRISE/IMaX. This confirms the conclusions of previous works (e.g., Graham et al. 2002). As a by-product, we have demonstrated the important effects of pure telescope diffraction on spectral profiles coming from the highly inhomogeneous, quiet-Sun photosphere.
Acknowledgements. We thank A. Vögler for kindly providing us with his MHD simulations. This work has been partially funded by the Spanish Ministerio de Educación y Ciencia, through projects ESP2006-13030-C0602, ESP2006-13030-C06-01, AYA2009-14105-C06-06, AYA2009-14105-C0603 , and by Junta de Andalucía through project P07-TEP-2687, including a percentage from European FEDER funds.

\section{References}

Álvarez-Herrero, A., Belenguer, T., Pastor, C., et al. 2006, Proc. SPIE, 6265 Barthol, P., Gandorfer, A. M., Solanki, S. K. et al. 2008, Ad. Space Res., 42, 70 Barthol, P., et al. 2010, Sol. Phys., in press [arXiv: 1009.2689]

Cabrera Solana, D., Bellot Rubio, L. R., \& Del Toro Iniesta, J. C. 2005, A\&A, 439, 687

Danilovic, S., Gandorfer, A., Lagg, A., et al. 2008, A\&A, 484, L17

Danilovic, S., Schüssler, M., \& Solanki, S. K. 2010, A\&A, 513, A1

Gandorfer, A. M., Solanki, S. K., Barthol, P., et al. 2006, Proc. SPIE, 6267, 25

Graham, J. D., López Ariste, A., Socas-Navarro, H., \& Tomczyk, S. 2002, Sol. Phys., 208, 211

Jochum, L., Collados, M., Martínez Pillet, V., et al. 2003, Proc. SPIE, 4843, 20

Khomenko, E. V., Shelyag, S., Solanki, S. K., \& Vögler, A. 2005, A\&A, 442, 1059

Kosugi, T., Matsuzaki, K., Sakao, T., et al. 2007, Sol. Phys., 243, 3

Lites, B. W., Elmore, D. F., \& Streander, K. V. 2001, Advanced Solar Polarimetry - Theory, Observation, and Instrumentation, 236, 33

Lites, B. W., Socas-Navarro, H., Kubo, M., et al. 2007, PASJ, 59, 571

Lites, B. W., Kubo, M., Socas-Navarro, H., et al. 2008, ApJ, 672, 1237 Martínez Pillet, V., et al. 2004, Proc. SPIE, 5487, 1152

Martínez Pillet, V., et al. 2010, Sol. Phys., in press [arXiv: 1009.1095]

Orozco Suárez, D. 2008, Ph.D. Thesis, Universidad de Granada

Orozco Suárez, D., \& del Toro Iniesta, J. C. 2007, A\&A, 462, 1137

Orozco Suárez, D., Bellot Rubio, L. R., del Toro Iniesta, J. C., et al. 2007, ApJ, 670, L61

Orozco Suárez, D., Bellot Rubio, L. R., Vögler, A., \& Del Toro Iniesta, J. C. 2010, A\&A, 518, A2

Paxman, R. G., Seldin, J. H., Loefdahl, M. G., Scharmer, G. B., \& Keller, C. U. 1996, ApJ, 466, 1087

Pietarila Graham, J., Danilovic, S., \& Schüssler, M. 2009, ApJ, 693, 1728

Ruiz Cobo, B., \& Del Toro Iniesta, J. C. 1992, ApJ, 398, 375

Sánchez Almeida, J., Ruiz Cobo, B., \& Del Toro Iniesta, J. C. 1996, A\&A, 314, 295

Sánchez Almeida, J., Emonet, T., \& Cattaneo, F. 2003, ApJ, 585, 536

Scharmer, G.B. 2006, A\&A, 447, 1111

Trujillo Bueno, J., Shchukina, N., \& Asensio Ramos, A. 2004, Nature, 430, 326 Tsuneta, S., Ichimoto, K., Katsukawa, Y., et al. 2008, Sol. Phys., 249, 167

van Noort, M., Rouppe van der Voort, L., \& Löfdahl, M. G. 2005, Sol. Phys., 228, 191

Vargas Domínguez, S. 2008, Ph.D. Thesis. Universidad de La Laguna

Vögler, A., \& Schüssler, M. 2007, A\&A, 465, L43

Vögler, A., Shelyag, S., Schüssler, M., et al. 2005, A\&A, 429, 335

$\overline{5}$ The study was carried out for the pair of Fe I lines at $630 \mathrm{~nm}$. 